\title{
Publisher's Note: Killing (absorption) versus survival in random motion
}

[Phys. Rev. E 96, 032104 (2017)]

Piotr Garbaczewski

(Q) (Received 12 February 2018; published 16 February 2018)

DOI: 10.1103/PhysRevE.97.029902

This paper was published online on 5 September 2017 with errors in Eqs (27) and (28). Equation (27) should read as

$$
\begin{aligned}
k(y, x, t) & =[\exp (-t \hat{H})](y, x)=\frac{1}{\sqrt{\pi}} \exp \left[-\left(x^{2}+y^{2}\right) / 2\right] \sum_{n=0}^{\infty} \frac{1}{2^{n} n !} H_{n}(y) H_{n}(x) \exp \left(-\epsilon_{n} t\right) \\
& =\exp (-t / 2)(\pi[1-\exp (-2 t)])^{-1 / 2} \exp \left[\frac{1}{2}\left(x^{2}-y^{2}\right)-\frac{\left(x-e^{-t} y\right)^{2}}{\left(1-e^{-2 t}\right)}\right] \\
& =\frac{1}{(2 \pi \sinh t)^{1 / 2}} \exp \left[-\frac{\left(x^{2}+y^{2}\right) \cosh t-2 x y}{2 \sinh t}\right] .
\end{aligned}
$$

Equation (28) should read as

$$
k(x, t \mid u, T) \sim \frac{1}{\sqrt{\pi} e^{(T-t) / 2}} e^{-\frac{1}{2}\left(x^{2}+u^{2}\right)}
$$

The equations have been corrected as of 6 February 2018. The equations are incorrect in the printed version of the journal. 